\title{
Developing A Mathematical Model For Locating Facilities And Vehicles To Minimize Response Time
}

Constance Lightner, (Email: clightner@uncfsu.edu), Fayetteville State University Asad Tavakoli, (Email: tavakoli@uncfsu.edu), Fayetteville State University Yahya Fathi, (Email: fathi@eos.ncsu.edu), North Carolina State University

\begin{abstract}
Traditional mathematical models for locating/allocating vehicles and facilities are reviewed and extended to illustrate how to formulate and solve a problem of minimized response time, given resource constraints. Results indicate that the average response time can be significantly improved through strategically allocating vehicles throughout the service area. Furthermore, the modified model was shown to outperform the traditional model as the number of vehicles allocated to a fixed number of facilities increase. Implications are identified for applications such public transit systems, wholesale and distribution operations.
\end{abstract}

\section{LITERATURE REVIEW}

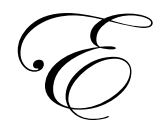

arly literature on location problems was based upon the traditional set covering problem. Set covering describes the minimum number of facilities needed to cover all system demand. A population is considered served, or covered, when a facility is sited within the maximal service distance. Church and Revelle [5] presented the Maximal Covering Location Problem (MCLP) to maximize the total amount of population served within a maximal service distance, given a fixed number of facilities. Under this objective, some population is left uncovered by the model. To address this problem Church and Revelle created the MCLP with mandatory closeness constraints and a bicriterion model that maximizes the amount of demand covered within the maximal distance and minimizes the distance traveled from the uncovered demand to its nearest facility, simultaneously [4].

Hakimi [12] developed a model similar to the MCLP termed the P-median problem. The P-median problem weights the distance between demand nodes and facilities by the associated demand quantity and calculates the total weighted travel distance between demand nodes and facilities. The model then seeks to find the location of $\mathrm{P}$ facilities so as to minimize the total demand weighted travel distance between demands and facilities. Additional research has been conducted to expanded upon the p-median approach to the emergency vehicle location problem and analyze sources of modeling errors [8,9]. These models offer techniques to improve the efficiency of the traditional p- median problem.

A review of the EMS system in Austin, Texas that relied upon MCLP based models exposed the necessity to construct alternative models that addressed the issue of vehicle availability. Daskin's [7] Maximal Expected Coverage Location Problem (MEXCLP) examines equipment availability within the context of location models. The objective of the MEXCLP is to locate emergency vehicles so as to maximize the expected coverage area, even in the event that multiple vehicles are in use. The model is based on the assumption that the probability that a randomly chosen vehicle is busy is independent of any other vehicle being in use. Bernardo and Repede [2] later modified this model to incorporate time varying demand and multiple states of vehicle availability.

Schilling et al. [17], developed the Tandem Equipment Allocation Model (TEAM) and Facility-Location, Equipment-Emplacement Technique (FLEET) model to allocate equipment with varying capabilities and demands, 
and possibly allocate the equipment and facilities simultaneously. The FLEET model has proven to be effective in the location-allocation of fire stations and equipment.

Bianchi and Church's [1] Multiple coverage, One-unit FLEET problem (MOFLEET) consolidates the MEXCLP and FLEET models by examining factors that must be addressed in ambulance location problems. This model minimizes the chances that an ambulance will not be available when demanded, by simultaneously allowing for coverage by alternative units. In multiple coverage models, a demand is defined as being completely covered when the demand area can be reached by at least $M$ vehicles within the specified time period. Gendreau [10,11] also addressed the multiple vehicle coverage issue by using a tabu search heuristic to determine optimal allocations.

Brotcorne, et. al. [3] recently compiled a comprehensive review of the 30 year history of ambulance location and relocation models. They categorized past models as follows: 1) Deterministic models which ignore stochastic considerations regarding the availability of ambulances; 2) Probabilistic models which reflect the fact that ambulances operate as servers in a queuing system and can sometimes be unavailable for calls; and 3) Dynamic models for relocating and deploying ambulances based upon real time data [11].

The models created by Daskin [7], Schilling, et. al [17], and Bianchi and Church [1] all address the possibility of an ambulance being busy when called, using a system wide busy probability. Revelle and Hogan [15] devised a method of estimating local busy probabilities using iterative and nonlinear methods. Their linear programming approach requires that a minimum number of vehicles be available in order for the problem formulation to be feasible.

Emergency service facility and location research has been expanded to incorporate various other systems with comparable characteristics. Other application areas have included: public transit systems, wholesale location and distributing operations and even reserve selection [14,16].

\section{MODEL SELECTION}

Several criteria were used in selecting an optimization model for the Cumberland County EMS system. The efficacy of the model was contingent upon several factors; namely:

- $\quad$ The model optimally and simultaneously locates facilities and allocates vehicles;

- $\quad$ The model considers the possibility that a vehicle may be unavailable when called;

- $\quad$ The model allows for multiple coverage;

- $\quad$ The model is efficient in terms of computing time; and

- $\quad$ The model generates results useful to laypersons.

The MOFLEET model discussed in the previous section satisfies these objectives and was adopted, with modifications, for this project.

The MOFLEET model has an objective function which seeks to minimize the expected amount of population not covered within a specified time period $t$. This function is based upon the system-wide parameter $\mathrm{u}$, the probability that a randomly selected vehicle will be unavailable when called. Original set covering models present a parallel between attaining this objective and our original goal of minimizing the response to answering demand $[5,6]$. The constraints designate $\mathrm{F}$ facility sites from a set of $\mathbf{J}$ potential locations and allocate $\mathrm{E}$ vehicles to the selected facilities. Additional constraints ensure that no more than $\mathrm{C}$ vehicles are located at a site and assist in penalizing the objective function value when nodes are not covered by at least $\mathrm{M}$ vehicles (the minimum number of vehicles required to provide full coverage to a node). The mathematical formulation of the MOFLEET model is provided in Appendix A. 


\section{MOLEET MODIFICATIONS}

Since the parameter $\mathrm{u}$ appears in the objective function, it is understandable that altering the value or method of computing u, could alter the model's results. Daskin [6] gives a formula (see first author's website for detailed formulation) for computing the parameter u based upon the average number of calls per day, the average service time per call, and the number of vehicles employed by the system.

The current model assumes an equal probability of a vehicle being busy across the system. This system-wide probability simplifies the model greatly; however, it is highly unlikely that a system will actually have a uniform system busy probability. Instead, we believe that there should be varying values for different demand centers in the system. Since it is unrealistic to expect vehicles in the inner city to have the same level of activity as vehicles located in remote, rural areas, we propose using local busy estimates to improve the model's performance. As such, we will use $\mathrm{u}_{\mathrm{i}}$ to denote the probability of a vehicle being busy at demand center $i$.

Our local busy probabilities, $\mathrm{u}_{\mathrm{i}}$, are based upon the amount of system demand in its local area. This local area is designated as all nodes located within $t$ minutes (as specified by the MOFLEET model). The $\mathrm{u}_{\mathrm{i}}$ values are computed as the product of the global u parameter and the ratio of the total local area demand of node $i$, to the average system local area demand.

In the MOFLEET model, the parameter $\mathrm{M}$ is used to specify the minimum number of vehicles required to provide full coverage to an area (see first author's website for detailed formulation). This value is computed utilizing our local busy probability values. Thus $\mathrm{M}_{\mathrm{i}}$ values are unique to each node.

The performance of the MOFLEET model is determined by computing the percentage of demand covered by the model's allocation of vehicles. Jayaraman and Srivastava [13] gives a formula for computing this measure (see first author's website for detailed formulation). We refer to their measure as MOFLEET CVG. We substitute our local $\mathrm{u}$ and $\mathrm{M}$ values into their formulas in order to evaluate our modified model, and we refer to this measure as Local CVG.

Our Modified MOFLEET model can be easily restated by substituting our $\mathrm{M}_{\mathrm{i}}$ and $\mathrm{u}_{\mathrm{i}}$ values in the original model in place of the global parameters. Although these changes may appear subtle, the integration of these local parameters with the original MOFLEET model alters the model's optimal sites to accommodate the dynamic demand patterns across the system.

\section{DATA COLLECTION AND ANALYSIS}

Cape Fear Valley Medical Center funded a project to determine optimal locations for ambulances and facilities in Cumberland County to minimize their response time. Their goal met the objective of the MOFLEET model. The data required for this model are compiled from the county emergency dispatch database (managed by Cumberland County Information Services) and EMS officials. Based on calls received, the county was divided into grids or "analysis zones". The structure of these analysis zones (nodes) is based on travel-time, population density, traffic flow, and other pertinent considerations. Following analysis of these factors, a network consisting of 119 zones covering the entire county was established. In effort to meet the national goal, our model was designed to minimize the amount of uncovered demand within eight minutes. Selecting eight as our target travel time sets the standard for determining the expected coverage that an optimal allocation will yield. Increasing or decreasing this value will respectively alter the expected coverage rendered by our model for a single vehicle distribution.

We analyzed the system to determine the eight-minute coverage for each zone. The accuracy of the travel distances was verified through several processes. First, an eight-minute coverage region was established for each node using a map of the county depicting major traffic arteries. Second, "test runs" were performed on established nodes using personal vehicles and travel with on-call ambulances. Third, estimated travel distances for each node were refined and verified by EMS paramedics. 
A sample of 14,130 EMS calls was used to determine the volume of demand in each node. The sample was divided into three groups; total call volume, day shift call volume and night shift call volume. The database included grid coordinates that were subsequently transformed to represent analysis zones. The distribution of average daily calls reveals some variation in demand. Despite these differences, the degree of variation did not warrant further partitioning of the data.

We analyzed the variation in demand and ran the model using the demand during various time periods. We compared the optimal allocations during different time periods and the optimal allocation using the average daily demand and noticed very little changes (in terms of coverage and optimal sites). Ultimately city officials decided against the option of redistributing vehicles to alternative stations throughout the day in order to experience slight coverage improvement. Thus the average daily volume of calls was used to represent node demand.

The city managers set $\mathrm{C}$ (the maximum number of vehicles to allocate to a single node) equal to 2 and $\mathrm{U}$ (the maximum number of vehicles that should be available to a coverage region) equal to 4 . The local $u_{i}$ and $M_{i}$ values were also computed using the appropriate formulas.

In summary, the following procedure was followed to collect the appropriate data for our model:

- $\quad$ Divide the service region into analysis zones;

- $\quad$ Specify $t$, the target time to respond to a call;

- $\quad$ Establish the coverage region for each zone, i.e., determine all zones that can be reached within $\mathrm{S}$ minutes from the respective zone, $\mathrm{N}_{\mathrm{i}}$;

- $\quad$ Specify the number of facilities, F, and vehicles, E, to allocate;

- $\quad$ Specify the set of potential facility locations, J;

- $\quad$ Determine the daily demand for each zone (daily demand is sufficient if significant changes do not exist throughout the day), $\mathrm{a}_{\mathrm{i}}$;

- $\quad$ Specify the maximum number of vehicles to allocate to a single node, $C$;

- $\quad$ Specify maximum number of vehicles that should be available to a coverage region, $U$;

- $\quad$ Compute $\mathrm{u}_{\mathrm{i}}$ for all zones; and

- $\quad$ Compute Mi, using formulas.

\section{EXPERIMENTAL RESULTS}

Two LINGO programs were written to implement the MOFLEET and Modified MOFLEET model formulations, presented in the previous section. We applied the Original and Modified MOFLEET Models to the 119 node network discussed in the last section to explore the changes that our Modified Model and Local CVG formula introduce. In order to have some measure of how well the underlying assumptions that govern these models perform, we first determined the MOFLEET and Local CVG values that the current vehicle sites provide. The city currently has six vehicles sited at nodes $32,34,41$ (2 vehicles), 58 , and 114 . Records indicate that $70 \%$ of the system's demand is covered within 10 minutes, using these vehicle sites (This suggests that considerably less than $70 \%$ of the calls are covered within 8 minutes). For these sites, MOFLEET CVG and Local CVG state that $74.5 \%$ and $46.3 \%$, respectively, of the demand should be covered within 8 minute time period. Thus our modified measure appears to give a more accurate portrayal of the actual coverage experienced by their system.

Next, we ran the models for varying values of $\mathrm{E}$ and $\mathrm{F}$, so that the corresponding levels of coverage could be assessed. In varying these values, one can determine whether the cost of operating additional facilities and vehicles is worth the increase in coverage. We expect these problems to reveal that our Modified MOFLEET model increases a system's percent of demand covered, when local busy estimates are considered in calculating the system's coverage percentage. Table 1 (Appendix B) displays the results of the Original MOFLEET model for varying numbers of facilities and vehicles. We evaluated the allocations given by the MOFLEET model using the MOFLEET CVG and Local CVG measures, and displayed the results in the last two columns of the table. Similarly, Table 2 (Appendix B) presents the results of the Modified MOFLEET model for varying numbers of facilities and vehicles. Notice from these tables that when the MOFLEET CVG formula is used to evaluate the coverage that the two models give for 
corresponding $\mathrm{F}$ and $\mathrm{E}$ values, the MOFLEET allocation gives higher coverage values. Similarly, when the Local CVG formula (coverage formula using $\mathrm{u}_{\mathrm{i}}$ values) is used to evaluate both models, the Modified MOFLEET model gives the highest coverage values. This result shows that the corresponding coverage formulas consistently evaluate the performance of the models.

Additionally, we observe that there is a large difference between the MOFLEET CVG and the Local CVG values for many of the instances presented on these tables. Moreover, we realize that the difference between these values decrease as E increases. This phenomenon suggests that MOFLEET's concept of global $\mathrm{u}$ and $\mathrm{M}$ parameters, overestimates the $\%$ of covered demand for smaller values of E. Our limited experience with the data of the City of Fayetteville supports the notion that the Local CVG values are closer to the observed values. Hence, we believe that Local CVG is a more realistic measure to evaluate system coverage. Therefore, based on the information presented in the tables, the Modified MOFLEET model is believed to be the better model because its Local CVG values are consistently higher than the optimal allocations given by the MOFLEET model.

Although we appreciate the changes that our Modified Model introduces to our system, we wish to recognize that this model does not completely disregard the optimal sites given by the MOFLEET Model. If drastic allocation changes existed for all problems, we would have been reluctant to quickly embrace and accept this new model. Instead, as presented on Tables 1 and 2, many of the optimal sites remain the same, or within the same vicinity. In fact, for $\mathrm{F}=8, \mathrm{E}=8$, the two models site 5 of 8 vehicles at the same locations, and for $\mathrm{F}=8, \mathrm{E}=10$, this fraction increases to 8 of 10 same location sites In essence, the Modified Model retained many of the vehicle locations (or within close proximity) given by MOFLEET, and altered the others to better accommodate the dynamics of the city, introduced by the incorporation of local parameters.

\section{INTERPRETING MODEL RESULTS AND DECISION MAKING}

The analysis performed in the last section was conducted to exhibit how our Modified MOFLEET Model enhances the original model's sensitivity to varying demand patterns across the city. This analysis can provide city officials with the necessary information to determine how to increase the city's percent of covered demand (within the 8 minute time constraint).

An interesting fact that Table 2 (Appendix B) reveals is that locating vehicles at additional facilities is not always optimal. This table shows for $\mathrm{F}=7, \mathrm{E}=8$ and $\mathrm{F}=8, \mathrm{E}=8$, the Modified model gives the same allocations. This result indicates, that operating 8 facilities, with 8 vehicles, does not increase coverage. Hence, if given 8 vehicles to allocate to the system, we should choose to operate only 7 facilities, with the 8 vehicles distributed among them, as given in Table 2. Additionally, the table shows how increasing the number of vehicles for a fixed value of $F$ impacts coverage. Although the increase in coverage may appear minimal in some cases, we must remember that even if the addition of a vehicle to the system only increases coverage by $1.5 \%$, during the course of a year this additional vehicle could affect approximately 230 calls. Using the tables presented in the Appendix, the officials can select an allocation that increases the city's coverage percentage, while optimizing the cost of achieving the desired level of coverage.

\section{CONCLUSIONS}

We have studied an approach for solving the Emergency Vehicle-Facility Location Problem using the MOFLEET mixed integer programming model with modifications. This approach is applicable to all systems that aim to reduce their response time for answering demand, when the possibility of vehicle unavailability exists. We provided detailed guidance for the data collection process required to implement such a model and obtain useful results. Model results were interpreted and discussed to show their usefulness in making critical system design decisions. The ultimate location-allocation decisions would depend upon the level of resources that the system employs upon considering all relevant costs.

The city re-allocated the six vehicles employed by the system in accordance with the recommendations provided in the tables of this research. Preliminary analysis shows some improvement in their average response time. Data is being collected to determine the actual percentage of calls covered within the eight minute target. We are 
reluctant to completely embrace the evaluation of coverage technique provided by Jayaraman and Srivastava [13] and modified in this research. We recognize the need for a uniform assessment technique, such as a simulation, for evaluating and comparing optimal allocations from proposed techniques. Future research will include sensitivity analysis on the parameter $\mathrm{u}$ to determine if small changes in estimating u will drastically alter optimal allocations. In addition, simulation models will be created to evaluate and compare a variety of approaches to solving our problem.

\section{REFERENCES}

1. G. Bianchi and R.L. Church (1988), A Hybrid Fleet Model for Emergency Medical Design, Social Sci. Med., 26, 163-171.

2. Bernardo, J. and J. Repede (1988), Two-tier Emergency Medical Service System: Analysis and Extension of Current Location Methodologies, Presented at the 1988 Annual Meeting of the Decision Sciences Institute, Las Vegas, NV.

3. Brotcorne, L., G. Laporte, and F. Semet (2003), Ambulance Location And Relocation Models, European Journal of Operational Research, 147/3, 451-463.

4. Church, R., J. Current, and J. Storbeck (1991), A Bicriterion Maximal Covering Location Formulation which Considers the Satisfaction of Uncovered Demand, Decision Sci., 22, 38-52

5. Church, R. and C. Revelle (1974), The Maximal Covering Location Problem, Papers Regional Sci., 32, 101118.

6. Daskin, M.S. (1982), An Application of an Expected Covering Model to Emergency Medical Service System Design, Decision Sci., 13, 416-439.

7. Daskin, M.S. (1983), A Maximal Expected Set Covering Location Model: Formulation, Properties, and Hueristic Solution, Transportation Science, 17, 48-69.

8. Erkut, E. and B. Bozkaya (1999), Analysis of Aggregation Errors for the P-Median Problem, Computers and Operations Research, 26, 1075-1096.

9. Francis, R.L., T.J. Lowe, and Arie Tamir (2000), Aggregation Error Bounds for a Class of Location Models, Operations Research, 48/2, 294-307.

10. Gendreau, M., G. Laporte, and F. Semet (2000), Solving an Ambulance Location Model by Tabu Search, Location Science, 5/7, 75-88.

11. Gendreau, M., G. Laporte, and F. Semet (2001), A Dynamic Model And Parallel Tabu Search Heuristic For Real-Time Ambulance Relocation, Parallel Computing, 27, 1641-1653.

12. Hakimi, S.L. (1964), Optimum Locations of Switching Centers and the Absolute Centers and Medians of a Graph, Operations Research, 12: 450-459.

13. Jayaraman, V. and Srivastava R. (1995), A service logistics model for simultaneous siting of facilities and multiple levels of equipment, Computers and Operations-Research, 191-204.

14. Love R.F., Morris J.G., and Wesolowsky, G.O. Facilities location: Models and Methods. New York: NorthHolland, 1988.

15. Revelle, C. and K. Hogan (1986), A Reliability Constrained Siting Model with Local Estimates of Busy Fractions, Environ. Planning, B 15, 143-152.

16. ReVelle, C., J.C. Williams, and J.J. Boland, (2002), Counterpart Models in Facility Location Science and Reserve Selection Science, Environmental Modeling \& Assessment. Vol. 7, No. 2, 71.

17. D. Schilling, D.J. Elzinga, J. Cohon, R. Church, and C. Revelle (1979), The TEAM/FLEET Models for Simultaneous Facility and Equipment Siting, Transportation Sci., 13, 163-175. 


\section{APPENDIX A: The MOFLEET Model}

The following model is adopted from Bianchi and Church [1]:

MINIMIZE $\quad \sum_{i \varepsilon I} \sum_{k=1}^{M}(1-u) u^{k-1} a_{i} y_{i k}$

Subject to :

$$
\begin{aligned}
& \sum_{j \varepsilon N_{i}} x_{j}+\sum_{k=1}^{M} y_{i k} \geq M \quad \text { For all } i \in J \\
& \sum_{j \varepsilon J} x_{j}=E \\
& \sum_{j \varepsilon J} z_{j}=F \\
& x_{j} \leq C z_{j} \quad \text { For all } j \in J \\
& x_{j} \geq 0 \quad \text { Integer for all } j \in J \\
& z_{j} \quad \text { and } \quad y_{i k}=\{0,1\} \text { For all } i, j \text {, and } k
\end{aligned}
$$

where:

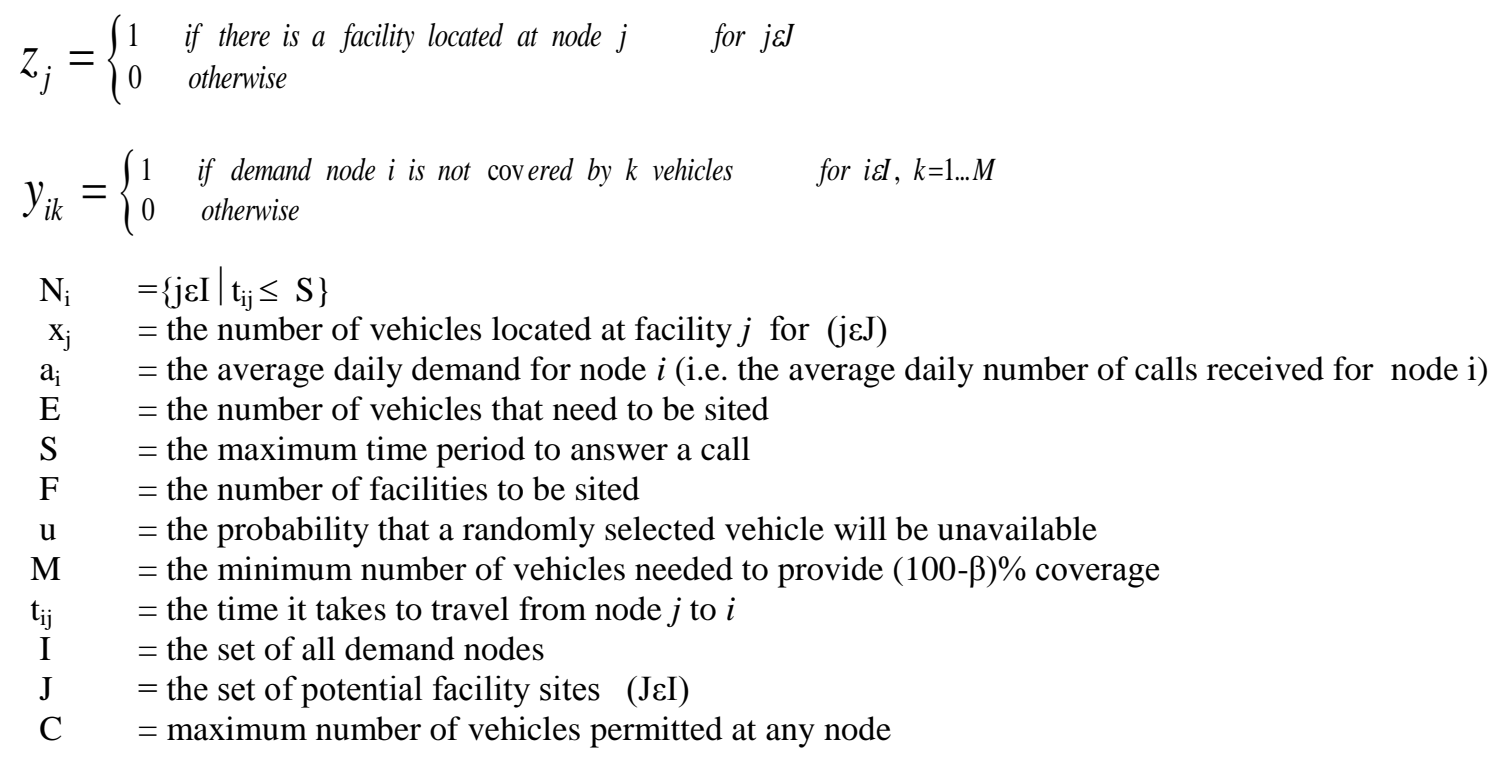


APPENDIX B: Model Results

Table 1: MOFLEET Model Results

\begin{tabular}{|c|c|l|c|c|}
\hline F & E & \multicolumn{1}{|c|}{ Optimal Vehicle Locations } & $\begin{array}{c}\text { \% of demand } \\
\text { covered (MOFLEET } \\
\text { CVG) }\end{array}$ & $\begin{array}{c}\text { \% of demand } \\
\text { covered (Local CVG) }\end{array}$ \\
\hline 6 & 6 & $19,26,32,34,55,57$ & 81.54 & 53.595 \\
\hline 6 & 8 & $5,26,41,41,44,44,47,94$ & 87.879 & 73.869 \\
\hline 6 & 10 & $19,19,26,34,34,54,54,57,57,94$ & 90.804 & 82.277 \\
\hline 7 & 8 & $5,26,41,41,44,44,47,94$ & 87.879 & 73.869 \\
\hline 7 & 10 & $19,19,26,34,34,54,57,57,94,101$ & 92.137 & 83.463 \\
\hline 7 & 12 & $19,19,26,26,34,34,54,54,57,57,94,101$ & 93.81 & 88.07 \\
\hline 8 & 8 & $19,26,32,34,55,57,94,101$ & 89.049 & 73.89 \\
\hline 8 & 9 & $19,19,26,34,45,55,57,94,101$ & 91.307 & 80.374 \\
\hline 8 & 10 & $5,26,31,31,47,47,55,57,94,101$ & 92.796 & 85.344 \\
\hline 8 & 11 & $19,19,26,34,34,54,57,57,63,94,101$ & 93.812 & 86.83 \\
\hline 8 & 12 & $19,19,26,34,34,54,54,57,57,63,94,101$ & 94.702 & 89.208 \\
\hline 8 & 13 & $19,19,26,26,34,34,54,54,57,57,63,94,101$ & 95.31 & 90.566 \\
\hline 8 & 14 & $19,19,26,26,34,34,54,54,57,57,63,94,94,101$ & 95.704 & 91.528 \\
\hline 9 & 10 & $5,26,31,31,47,47,55,57,94,101$ & 92.796 & 85.344 \\
\hline 9 & 12 & $19,19,26,34,34,41,57,57,63,64,94,101$ & 95.034 & 99.974 \\
\hline 9 & 14 & $13,19,19,19,26,26,45,45,55,55,63,64,94,94,101$ & 96.143 & 92.174 \\
\hline
\end{tabular}

Table 2: Modified MOFLEET Model Results

\begin{tabular}{|c|c|l|c|c|}
\hline $\mathbf{F}$ & $\mathbf{E}$ & \multicolumn{1}{|c|}{ Optimal vehicle locations } & $\begin{array}{c}\text { \% of demand } \\
\text { covered (MOFLEET } \\
\text { CVG) }\end{array}$ & $\begin{array}{c}\text { \% of demand } \\
\text { covered (Local CVG) }\end{array}$ \\
\hline 6 & 6 & $31,31,34,44,55,57$ & 79.013 & 57.097 \\
\hline 6 & 8 & $26,31,31,34,44,55,57,57$ & 85.151 & 78.24 \\
\hline 6 & 10 & $5,26,31,31,45,45,55,55,70,70$ & 89.332 & 85.602 \\
\hline 7 & 8 & $26,31,31,34,44,55,57,94$ & 87.49 & 78.575 \\
\hline 7 & 10 & $5,26,31,31,44,44,47,55,55,94$ & 91.114 & 88.034 \\
\hline 7 & 12 & $5,5,26,31,31,47,47,55,55,57,57,94$ & 92.918 & 90.771 \\
\hline 8 & 8 & $26,31,31,34,44,55,57,94$ & 87.49 & 78.575 \\
\hline 8 & 9 & $5,26,31,31,44,47,55,57,94$ & 90.683 & 84.73 \\
\hline 8 & 10 & $5,26,31,31,44,47,55,55,57,94$ & 91.844 & 88.632 \\
\hline 8 & 11 & $5,26,31,31,44,44,47,55,55,94,101$ & 93.427 & 91.342 \\
\hline 8 & 12 & $5,26,31,31,44,44,47,55,55,94,94,101$ & 94.255 & 92.747 \\
\hline 8 & 13 & $5,5,26,31,31,47,47,55,55,57,57,94,101$ & 95.129 & 93.603 \\
\hline 8 & 14 & $5,5,26,26,31,31,47,47,55,55,57,57,94,101$ & 95.625 & 94.287 \\
\hline 9 & 10 & $5,26,31,31,44,47,55,57,94,101$ & 93.106 & 88.95 \\
\hline 9 & 12 & $5,26,31,31,44,44,47,54,54,57,94,101$ & 94.548 & 93.38 \\
\hline 9 & 14 & $5,26,26,31,31,34,34,55,55,57,57,63,94,101$ & 96.098 & 94.209 \\
\hline
\end{tabular}

